\title{
Papers Presented at the Annual Meetings of the Hip Society 2011
}

\section{Editorial Comment}

\author{
Stuart B. Goodman MD, PhD
}

Published online: 15 October 2011

(C) The Association of Bone and Joint Surgeons (R 2011

This symposium is composed of selected papers delivered at the Fall 2010 Closed Meeting of the Hip Society in New York, NY, USA, and the Open Scientific Meeting of the Hip Society in conjunction with the 2011 Annual Meeting of the American Academy of Orthopaedic Surgeons in San Diego, CA, USA. The focus of these meetings was on contemporary topics related to alternative bearing surfaces and novel prosthesis designs for primary hip arthroplasty, complications associated with primary and revision hip arthroplasty, surgical strategies to deal with extensive bone loss during revision surgery, and hip joint-preserving procedures.

With the advent of alternative bearing surfaces, including the use of larger femoral heads, it is hoped implant longevity will be improved and complications such as periprosthetic osteolysis and recurrent dislocation will be mitigated. For metal-on-crosslinked polyethylene, these expectations appear to be true, at least in the intermediate term. The same could be said of ceramic-on-ceramic bearings, although smaller femoral heads $(28-32 \mathrm{~mm})$ are currently used and optimal implant position is crucial. The more controversial articulation is metal-on-metal, the use of which has dramatically decreased globally over the last several years due to concerns about adverse tissue reactions and metal ion release. Nevertheless, some metal-on-metal implants are functioning well in the short and intermediate

All ICMJE Conflict of Interest Forms for authors and Clinical Orthopaedics and Related Research editors and board members are on file with the publication and can be viewed on request.

S. B. Goodman $(\square)$

Department of Orthopaedic Surgery, Stanford University

Medical Center Outpatient Center, 450 Broadway St.,

M/C 6342, Redwood City, CA 94063, USA

e-mail: goodbone@stanford.edu

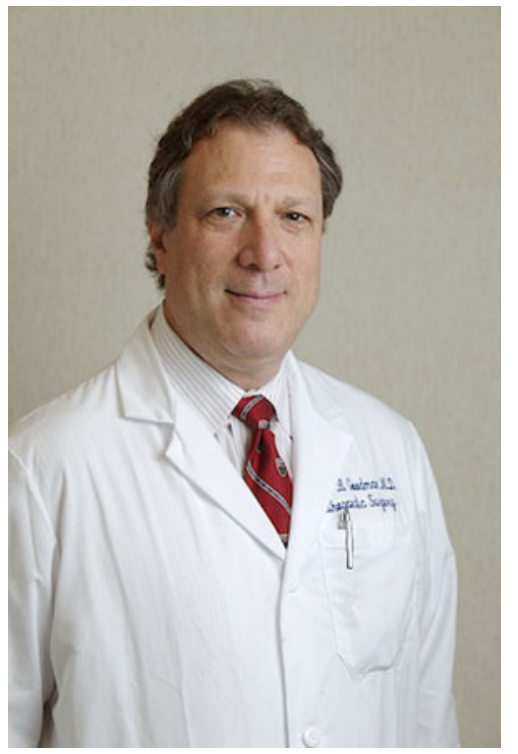

Fig. 1 Dr. Stuart B. Goodman is shown

terms. Patient selection and implant design, as well as precise surgical implantation, are key factors in durability. The shorter, metaphyseal-fitting femoral component for stemmed THA is another innovation that has achieved short-term success. Longer followup is necessary to properly assess the role of these new technologies.

Complications associated with primary and revision hip arthroplasty are still major problems for the patient and healthcare team. Although dislocation has become less common, new methods for imaging and assessing the amount of abduction and anteversion of the components have been described. The concept of "combined anteversion" has been proposed, that is, the sum of anteversion of both acetabular and femoral components. This may allow 
slightly more flexibility in the positioning of either component separately, which can be compensated when orienting the companion component. Novel techniques for closure of the capsule may further decrease the dislocation rate especially for hips approached posteriorly. Detailed algorithms for assessing and treating pulmonary embolism and optimizing anticoagulation are now enumerated, so that these conditions can be managed in a safe and effective manner.

Extensive loss of bone stock is a difficult challenge when dealing with cases demonstrating severe acetabular protrusion, implant loosening and osteolysis, periprosthetic fracture, and chronic infection. Longer-term followup has documented the success of impaction grafting of the acetabulum. The use of porous metallic augments, specialized custom implants, and other surgical constructs has recently been described for more extensive bone loss. Long-term followup is needed to assess the durability of these novel approaches.
Finally, joint-preserving operations continue to increase in number and include hip arthroscopy, reorienting pelvic osteotomy, and novel surgical treatments for osteonecrosis of the femoral head. The current challenge is to select the right patient for the right operation and to carry out the procedure meticulously to delay or possibly obviate the need for joint arthroplasty. These operations are often demanding with long learning curves. Future evidencebased studies will help determine the indications and outcomes for these newer operations.

This symposium is composed of basic and clinical research studies reported by members of The Hip Society as an educational tool for surgeons engaging in surgery of the hip. The members have presented their research in a standardized format that has undergone rigorous peer review. It is hoped this symposium will help guide the hip surgeon to improve patient care. On behalf of The Hip Society, we are pleased to present this information for your consideration. 